\title{
EL ENGAGEMENT Y SU IMPACTO EN EL SOCIAL MEDIA DEL ECUADOR
}

\section{ANALYSIS OF ENGAGEMENT IN THE SOCIAL MEDIA FROM ECUADOR}

\author{
Edgar Salas Luzuriaga ${ }^{1}$
}

\author{
Palabras clave: \\ Redes sociales, \\ plataforma, \\ influencia, \\ estrategia, \\ compromiso, \\ comunicación, \\ mercadeo, era \\ digital
}

\author{
Keywords: \\ Social networks, \\ platform, \\ influence, \\ strategy, \\ engagement, \\ communication, \\ marketing, digital \\ era
}

\begin{abstract}
Resumen
El presente estudio pretende analizar la incidencia que existe entre los usuarios ecuatorianos y su nivel de influencia al usar determinadas redes sociales, examinar el engagement de las respectivas plataformas sociales y analizar la razón de su utilización, siendo Facebook, Instagram y Snapchat las redes más usadas por la población ecuatoriana, no solo para el ámbito social, sino también para el ámbito laboral, destacándose así los emprendimientos nacionales.
\end{abstract}

Códigos JEL: M30, M31

\footnotetext{
${ }^{1}$ Director Académico de la Facultad de Comunicación. Universidad de Especialidades Espíritu Santo, Km. 2,5 vía a Samborondón, Ecuador E-mail: esalas@uess.edu.ec
}

\begin{abstract}
The present study aims to analyze the incidence that exists among Ecuadorian users and their level of influence when using certain social networks, examine the engagement of the respective social platforms and analyze the reason for their use, being Facebook, Instagram and Snapchat the most used networks by the Ecuadorian population, not only for the social sphere; but also for the work environment, highlighting national ventures.
\end{abstract}




\section{INTRODUCCIÓN}

Hoy el Marketing es una conversación y las marcas deben unirse a ella (Rosales, 2010). Las redes sociales hoy en día son parte de la vida cotidiana a nivel profesional como personal, además que cada vez son más usadas como canal de comunicación, por lo tanto, es importante poder entender su finalidad (Valerio, Herrera-Murillo, y Rodríguez-Martinez, 2014). Son plataformas virtuales vía internet donde los usuarios pueden publicar contenido sobre ellos y compartirlo (Goldsmith, Pagani y Lu, 2013). Hay distintas maneras de demostrar que existe engagement en las redes sociales ya que este se puede medir a través de: likes, compartidos, menciones, Facebook feelings, clicks, visitas, comentarios y reacciones. Cada interacción en cada red social tiene un peso y valor diferente para cada persona ya que las usan con diversos fines (Obeso, 2017).

En mercadotecnia, el engagement se denomina al grado de compromiso o fidelidad que pueda existir entre las personas y el contenido exhibido, quiere decir que mientras exista mayor identificación emocional por parte de los usuarios habrá más conexión y fidelidad con la marca. Se mide a través del nivel de identificación y la conexión emocional, es decir hay que pensar en la audiencia al momento de compartir publicaciones; que lleguen a ellos y no solamente para que el contenido sea compartido, sino también usando el humor para divertir a los seguidores, llamar a la acción a través de publicaciones, entre algunas otras acciones (Parra, 2017).

Por otro lado, a través del engagement se puede lograr identificar cuáles tácticas funcionan y cuáles no, medir el cumplimiento de objetivos y poder compararte con los competidores (Obeso, 2017). El objetivo de una marca siempre va a ser lograr un buen engagement $\mathrm{y}$ mejorarlo constantemente para así a futuro poder garantizar el éxito de la campaña publicitaria o esfuerzo de marketing de contenidos en el mercado.

El engagement es un elemento clave que hace que las personas escuchen y sigan tus consejos o recomendaciones, es decir que es lo que genera credibilidad y puede tener una gran influencia sobre los seguidores. Es considerado como uno de los 10 elementos principales que una estrategia de redes sociales debería tener (Redactor Rock Content, 2018).

En comparación con un estudio realizado en España, las redes sociales alcanzan el $81 \%$ de la población de entre 16 y 55 años (25.4 millones). Así, según el estudio anual de redes sociales publicado por IAB Spain, el $47 \%$ de los usuarios las utilizan para mantenerse informados $\mathrm{y}$, hasta en un 36\% para conocer productos y/o servicios de la marca (2016b). De igual manera se dice que WhatsApp y Facebook son las que más les gusta a los usuarios seguidos de Twitter, YouTube e Instagram.

El presente trabajo tiene como objetivo general el determinar la importancia y funcionalidad de las redes sociales en el diario vivir de las personas ecuatorianas, así también analizar la importancia de las redes sociales en la vida de los ecuatorianos e identificar las funciones de las redes sociales.

Las redes sociales se han convertido en una plataforma idónea para dar a conocer y aumentar la visibilidad de una marca o empresa, además de que también sirve para interactuar y analizar las acciones de los seguidores de estas. La presente investigación se enfocará en determinar el engagement de las redes sociales en el consumidor ecuatoriano, se busca además especificar cuáles son los canales por los que más se consumen, cuáles son los grupos demográficos que muestran más interés hacia ellas, las redes preferidas entre los consumidores y su razón del por qué. Este trabajo permitirá mostrar el análisis 
detallado del consumo de cómo cada una de las redes sociales y como estas influyen en el perfil nacional.

La comprensión profunda de estos datos nos facilitará aclarar los conceptos y los procesos que se llevaron a cabo con el fin de persuadir a la población del uso de estas redes. Este estudio puede permitir distinguir con claridad las potencialidades que cada una de estas plataformas representan para la comunicación. Esto posibilita no solo aumentar la eficiencia en relación a los futuros proyectos publicitarios, sino planificar con mejores resultados las estrategias publicitarias en los proyectos de campaña digital.

\section{REVISIÓN TEÓRICA}

Para entender mejor este mercado de campañas publicitarias hay que analizar el uso de las redes sociales. El acceso a internet en los hogares ecuatorianos es un indicador que ha ido creciendo rápidamente en los últimos años, en el 2012 apenas el $22 \%$ de los hogares accedía a internet, pero ese indicador fue subiendo con el paso del tiempo, hasta que para el 2016, llego a 36\%, es decir casi más cuatro de diez hogares ecuatorianos tiene acceso a este servicio, para el 2019 la cifra ha de ser mayor por la tendencia al alza.

También ha crecido el porcentaje de personas que utilizan internet al año 2016, $55.6 \%$ de ecuatorianos habían accedido a la web, los que más acceden a internet son los jóvenes entre 16 y 24 años, pues el $83 \%$ de ellos ha entrado a internet. "El mercado publicitario en Guayaquil se ha visto muy afectado desde la aparición de las redes sociales y las nuevas tecnologías de la información" (Interactive Advertising Bureau, 2019). Estas han logrado hacer que las agencias de publicidad en el país se actualicen y logren adaptarse al público objetivo, un público que se encuentra en las redes sociales.

El 2017 se vio definido por el mando y aumento de las redes sociales por sus aspectos multifuncionales que están permitiendo que los usuarios accedan a varios servicios, solo al usar un mismo medio de red social. Facebook implementó su peculiaridad de historias y permitió almacenar contenido preferido para sus usuarios, igualmente que la red de Instagram. Los dispositivos móviles se han comisionado del uso de redes sociales. Al peso, la totalidad de usuarios de smartphones usan Facebook, Instagram, Snapchat, Pinterest y LinkedIn y estas las utilizan a partir del dispositivo móvil.

De esta totalidad de usuarios, los crecidamente ávidos usuarios de celular son los usuarios de Facebook e Instagram. "El 2017 asimismo registra un acrecentamiento en el uso de redes sociales fotográficas como Instagram" (Interactive Advertising Bureau, 2019).

Este medio de red social permite el desplazamiento de contenido en fotografías generado por sus mismos usuarios, esto ha repuntado en aproximadamente todos los grupos demográficos, fundamentalmente en los usuarios lozanos o jóvenes, y todo gracias al adelanto progresivo del acceso a internet en los últimos años. Esta evolución en la conectividad, tiene que ver con qué; tres de cada diez hogares tienen una computadora de escritorio o portátil y nueve de cada diez tiene un teléfono celular.

En Ecuador, en marzo del 2017, habían 14.9 millones de líneas de celulares activas, de este alrededor de 85 millones corresponden a smartphones, es decir teléfonos celulares que tiene accesos a aplicaciones por internet como mails y redes sociales. "Esta situación ha dado fundamento a muchos fenómenos en el mercado publicitario $\mathrm{y}$ en específico a 
muchas nuevas ideas, el uso de estas ha afectado la producción" (Contreras, 2016, pág. 73).

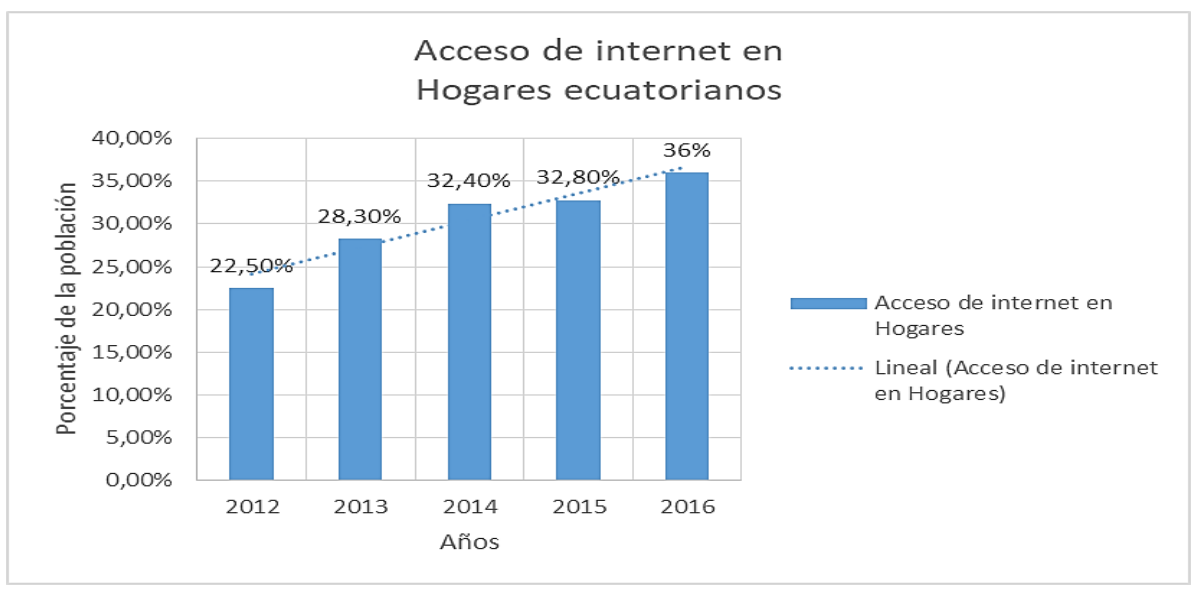

Figura 1. Acceso de internet_hogares ecuatorianos

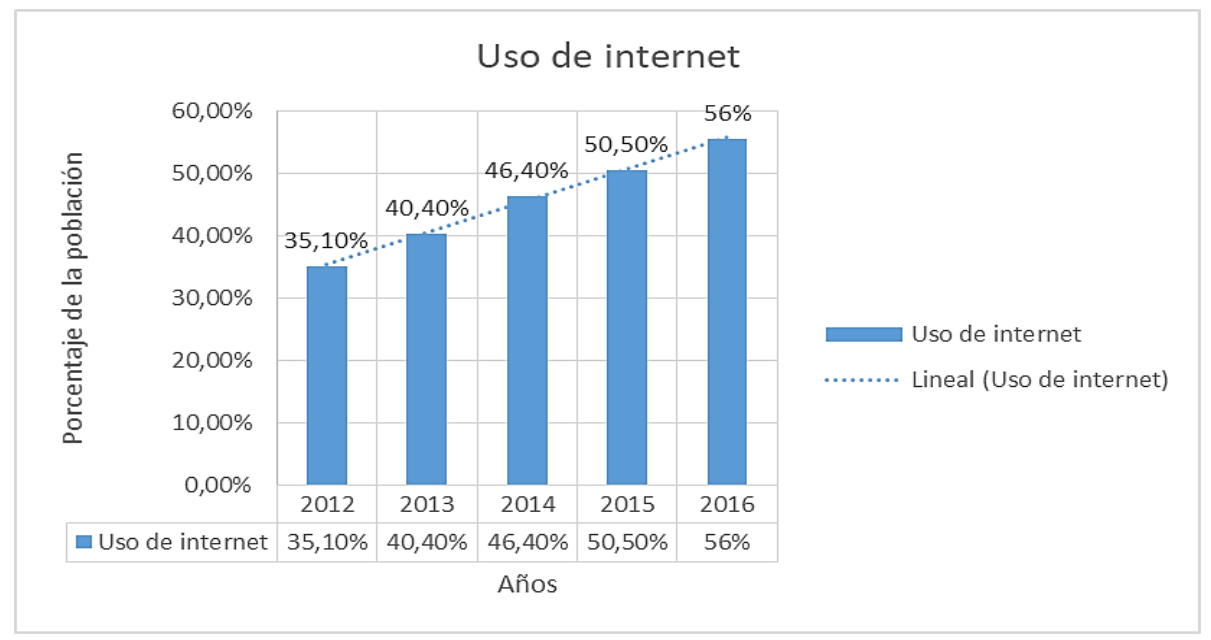

Figura 2. Uso de internet_Mercado publicitario Guayaquil

Al generar nuevos puestos de gestión y darles la posibilidad a nuevos emprendimientos en especial a las nuevas Agencias Digitales, de medios Sociales y Estudios de Diseño, quienes han percibido una ocasión para desarrollarse y les han dado uso. También al constar estas modalidades; un excedente de agencias pequeñas, estudios creativos y freelancers se generó un aumento de la competencia en la industria. Los freelancers, que en algunos casos solamente han realizado cursos por internet gratuitos $\mathrm{o}$ lo han aprendido relacionado a la jornada, tienen bajos costos de operaciones y terminan por conceder trabajos de baja particularidad a precios estrechamente bajos, lo que ha afectado a las agencias pequeñas y medianas que tienen gastos de realización crecidamente altos.

La situación de este consumo de canales para las agencias de publicidad tradicionales así mismo ha sentido los efectos de este acontecimiento, ya que en ellas se han elaborado los conocidos Departamentos Digitales, en los que se han asociado una sucesión de profesionales en el espacio publicitario e inclusive la inversión publicitaria de estos medios se han desarrollado de modo vertiginoso. 
Según el INEC, el principal uso que se da a internet es para obtener información, seguido por la comunicación en general, la educación y razones de trabajo. Sin embargo, una encuesta realizada a 331 millennials de Ecuador, revela que el acceso a redes sociales, es la principal actividad que este grupo poblacional realiza, en internet, en comparación, por ejemplo; mandar mails, hacer llamadas, ver videos, escuchar o leer noticias.

Una red social es una estructura capaz de comunicar entre sí a personas o instituciones. A través de internet se pueden establecer relaciones que creen grupos o comunidades con intereses comunes. Las redes sociales suponen un contacto ilimitado y a tiempo real. Esto se consigue gracias a la interactividad, uno de sus rasgos más distintivos y novedosos de las mismas.

Tiene como ventajas el reencuentro con conocidos, oportunidad de integrarse, propiciar contactos afectivos nuevos como búsqueda de pareja, amistad o compartir intereses, compartir momentos especiales con las personas cercanas a nuestras vidas eliminando fronteras geográficas conectando gente sin importar la distancia, establecer conexiones en el mundo profesional, tener información actualizada y por último permite comunicarnos en tiempo real. También tiene desventajas como la violación de la privacidad, exposición de vida privada, suplantación de personalidad, falta en el control de datos, pueden ser adictivas y devorar gran cantidad de nuestro tiempo y pueden apoderarse de todos los contenidos que publicamos.

\section{METODOLOGÍA}

A fin de evaluar la relación entre el engagement hacia las redes sociales y las razones de uso y participación de sus usuarios; Esta investigación adopta un desarrollo teórico que explora la naturaleza del comportamiento del engagement en un entorno virtual. El núcleo de este marco conceptual reside en la correspondencia entre la función de las redes sociales, como mecanismo de comunicación y el rol de la motivación en la elección entre redes alternativas (Smock, Ellison, Lampe, y Wohn, 2011). Del cual se desprende, una notable distinción entre las fuentes de uso y de participación en un contexto de red social; Sugiriendo específicamente, que su uso está orientado en todo tiempo y lugar hacia objetivos, y que la participación de la audiencia es un antecedente importante del engagement (Sun, Rubin, y Haridakis, 2008). De modo que, este trabajo postula a la motivación inherente en el uso de una red social, y al nivel de participación en la red, como precursores claves del grado de engagement de los usuarios.

Las principales motivaciones subyacentes al uso de redes fueron extraídas del Modelo de Contenido de las Redes Sociales (MCRS) desarrollado por Dolan, Conduit, Fahy, y Goodman, (2016). Este enfoque recurre a la Teoría de Uso y Gratificación (TUG) para predecir comportamientos específicos como resultado de las motivaciones en el uso de plataformas sociales (Smock, Ellison, Lampe, y Wohn, 2011). La construcción sugiere que el contenido de las redes sociales se puede clasificar en cuatro grupos principales; en función de su nivel de información (Cvijikj y Michahelles, 2013), entretenimiento (de Vries, Gensler, y Leeflang, 2012), remuneración (Lee, Hosanagar, \& Nair, 2018) y contenido relacional (Muntinga, Moorman, y Smit, 2011). La entrega de estas diversas formas de contenido, se espera, gratifique los motivos de los usuarios, intensificando las expresiones de Engagement.

Las cuatro variables de contenido se midieron a través del Cuestionario de Uso y Gratificación (CUG) desarrollado por Ludovic, Baghestan, y Sazmand (2013). El cuestionario consta de 11 declaraciones que son evaluados utilizando una escala de tipo 
Likert de cinco puntos que va desde "totalmente en desacuerdo" hasta "Totalmente de acuerdo". Para medir el engagement hacia las redes, se aplicó el "Cuestionario de participación en Redes sociales" (CPRS) propuesto por Przybylski, Murayama, DeHaan, y Gladwell (2013), que evalúa el engagement a través de preguntas sobre el uso de las redes sociales durante una semana, antes y después de que el participante incurra en situaciones de rutina como son dormir y desayunar; el cuestionario consta de cinco items con una escala Likert de 7 puntos que va desde "Ningún día" hasta "Todos los días". La participación del usuario dentro de las redes sociales fue aproximada a través de la frecuencia de uso diario, medido en horas. Esta valoración recoge la diferencia entre el uso en búsqueda de objetivos y el uso como reflejo de participación. La primera denota un uso poco frecuente y la segunda denota afinidad intrínseca, que recae en el uso prolongado. Luego, a la unificación de estos cuestionarios en un formato único, se incluyeron preguntas demográficas de edad y género que sirvieron como variables de control.

Las variables van a ser divididas en variables cualitativas y cuantitativas. En las variables cualitativas se analizará frecuencia y porcentaje, y su distribución calculada mediante el método del Chi cuadrado. En las variables cuantitativas se sacará una media y su distribución será dada por una desviación estándar. Estas variables van a ser analizadas mediante el programa de SPSS para Windows 10, previa ponerlas en Microsoft Excel para su posterior tabulación.

Los datos se obtuvieron de una muestra de las ciudades de Guayaquil y Quito. Los cuestionarios se distribuyeron en ambas ciudades durante un período de cuatro semanas en el primer trimestre del 2019. Dado que este estudio fue enfocado hacia usuarios de redes sociales, antes de la aplicación de los cuestionarios se les consulto a los participantes si poseían al menos una cuenta en una red social, si la respuesta era afirmativa, se continuaba con el procedimiento. Este proceso produjo una cohorte representativa a nivel terciario de 112 participantes, de los cuales, 69 son hombres y 43 son mujeres, con edades comprendidas entre 19 y 25 años, que representa la edad promedio de los usuarios de redes sociales más activos.

Ahora, para evaluar el impacto de la motivación inherente en el uso de una red social y el nivel de participación sobre engagement hacia las redes sociales, se formuló el siguiente modelo:

$$
E_{i}=\delta U_{i}+\theta P_{i}+u_{i}
$$

En donde $U$ es una matriz que contiene todos los factores motivantes asociados al uso de una red social, representados por las principales características de su contenido que son: la Información, el Entretenimiento, la Remuneración y el contenido Relacional; $P_{i}$ es un vector que representa el nivel de participación en redes, medido por el número de horas al día que el usuario interactúa en la red social; $E_{i}$ refleja el grado de engagement de los usuarios de redes sociales, y $u_{i}$ refleja el término de error o perturbación del sistema. Los parámetros asociados al modelo de regresión serán estimados por medio del método de mínimos cuadrados ordinarios con errores estándares robustos y con un nivel de confianza del $95 \%$.

\section{RESULTADOS}

La tabla 1 muestra un resumen de los factores propuesto como determinantes del engagement hacia las redes. Se presenta la media y el coeficiente de variación como criterio de comparabilidad del conjunto de variables. Adicionalmente se muestra la matriz de correlaciones con el fin de corroborar el supuesto de independencia entre las variables de respuesta. 
La variable Información presenta un nivel de dispersión mayor con respecto a las demás variables, con un coeficiente de variación de 0.24. El contenido Relacional es la que menor variación presenta, con un valor de 0.11. La Edad promedio de los participantes es de 22 años y el promedio al día de uso de redes sociales alcanza las cuatro horas. Por otra parte, el grado de asociación lineal entre las variables no supera el 10\%, lo que garantiza estimaciones robustas de los parámetros del modelo propuesto. Un dato interesante, es que la edad tiene una relación negativa con el entretenimiento y con el contenido relacional, sugiriendo un menor interés en estos aspectos a medida que la edad del usuario avanza.
Previa estimación de la ecuación propuesta, se efectuó la estandarización de todas las variables con el fin de ajustar las distintas medidas a una escala común y así, dejar la interpretación de los coeficientes en términos de desviaciones estándar, que facilite la comparabilidad de los efectos. Se estimaron un total de cuatro modelos. En el modelo 1 se incluyó únicamente las variables propuestas por la Teoría de Uso y Gratificación (TUG). En el modelo 2 se introduce la variable de "Tiempo en redes sociales" aproximada por las horas al día de uso de las redes sociales. En los dos modelos restantes se añadieron privativamente las variables de control; Edad y Género. La tabla 2 muestra los resultados obtenidos.

Tabla 1

Estadística descriptiva, coeficiente de variación e intercorrelaciones por factores

\begin{tabular}{|c|c|c|c|c|c|c|c|c|}
\hline \multirow{2}{*}{ Variable } & \multirow{2}{*}{$\mathbf{M}$} & \multirow{2}{*}{$\mathbf{C V}$} & \multicolumn{6}{|c|}{ Correlación } \\
\hline & & & 1 & 2 & 3 & 4 & 5 & 6 \\
\hline 1. Información & 12.21 & 0.240 & - & & & & & \\
\hline 2. Entretenimiento & 9.04 & 0.201 & 0.0384 & - & & & & \\
\hline 3. Remunerativo & 6.29 & 0.137 & 0.0468 & -0.0846 & - & & & \\
\hline 4. Relacional & 5.98 & 0.114 & 0.0985 & 0.0818 & 0.0615 & - & & \\
\hline 5. Edad & 22 & 0.188 & 0.0571 & -0.0617 & 0.0689 & -0.0946 & - & \\
\hline 6. Tiempo en redes sociales & 4.71 & 0.226 & 0.0539 & 0.0332 & 0.098 & 0.0497 & 0.0367 & - \\
\hline
\end{tabular}

\section{Tabla 2}

\section{Modelos de Regresión Estandarizada}

\begin{tabular}{|c|c|c|c|c|c|}
\hline Modelo teórico & Variable & 1 & 2 & 3 & 4 \\
\hline \multirow{8}{*}{$\begin{array}{l}\text { Perspectiva de Uso y } \\
\text { Participación }\end{array}$} & Información & $0.107 * *$ & $0.137 * *$ & $0.128^{*}$ & $0.107 *$ \\
\hline & & $(0.176)$ & $(0.178)$ & $(0.178)$ & $(0.183)$ \\
\hline & Entretenimiento & $0.131 * *$ & $0.150 * *$ & $0.167 * *$ & $0.171 * *$ \\
\hline & & $(0.224)$ & $(0.228)$ & $(0.233)$ & $(0.453)$ \\
\hline & Remunerativo & 0.092 & 0.070 & 0.067 & 0.097 \\
\hline & & $(0.224)$ & $(0.227)$ & $(0.230)$ & $(0.234)$ \\
\hline & Relacional & 0.114 & 0.129 & 0.084 & 0.097 \\
\hline & & $(0.231)$ & $(0.232)$ & $(0.234)$ & $(0.233)$ \\
\hline \multirow{6}{*}{ Control } & Tiempo en redes sociales & - & $0.164 *$ & $0.151 *$ & $0.161 *$ \\
\hline & & - & $(0.206)$ & $(0.441)$ & $(0.121)$ \\
\hline & Edad & - & - & -0.061 & -0.058 \\
\hline & & - & - & $(0.240)$ & $(0.239)$ \\
\hline & Género & - & - & - & 0.081 \\
\hline & & - & - & - & $(0.206)$ \\
\hline \multirow{2}{*}{ stadísticos de resumen } & ESR & 4.78 & 4.81 & 4.94 & 4.97 \\
\hline & $\mathrm{R}$ cuadrado ajustado & 0.283 & 0.329 & 0.269 & 0.228 \\
\hline
\end{tabular}

Nota: $* p<.1 ; * * p<.05 ; * * * p<.01$ 
En las cuatro últimas columnas se encuentran las estimaciones de los modelos corridos para cada una de las variables, con su respectivo error estándar. Las últimas dos filas muestran los estadísticos de resumen; particularmente el $\mathrm{R}$ cuadrado ajustado, que servirá como criterio de evaluación de ajuste entre modelos.

Con respecto a las variables de Uso y Participación; Existe evidencia estadística para afirmar un efecto positivo de la Información como razón de uso de una red social sobre el engagement, con un nivel de significancia del $5 \%$ en los modelos 1 y 2 , e inferior al $1 \%$ en los dos modelos restantes. Sugiriendo, que el deseo de buscar información es un factor motivador que conduce a las personas a mantenerse activos en una red social. De igual forma, el Entretenimiento en los cuatro modelos estimados presenta un impacto positivo y significativo sobre el nivel de engagement de los usuarios, con un nivel de significancia del 5\%. Resaltando el valor del entretenimiento en una plataforma de red social como una razón importante por la que los consumidores lo adoptan. El tiempo en redes sociales también presenta un impacto significativo en los tres modelos en los que fue incluido, con un nivel de significancia de $1 \%$. Las demás variables no presentan un efecto sobre el engagement.

El modelo 2 es el que mejor ajuste presenta, explicando un $33 \%$ de la variabilidad total del "engagement hacia las redes sociales". En ese orden, un incremento de una desviación estándar en las variables: Información, Entretenimiento y Tiempo en redes sociales, produce un impacto de, $0.13,0.15$ y 0.16 desviaciones estándar en el nivel de engagement del usuario promedio, respectivamente. La ventaja del uso de coeficientes estandarizados es la posibilidad de comparar los efectos en términos de importancia relativa. En otras palabras, los hallazgos presentan al Tiempo en redes sociales, identificado como medida de participación en redes sociales, como el factor que mejor predice el grado de engagement, por sobre los motivos inherentes al uso de la red.

\section{CONCLUSIONES}

Las Redes Sociales son mecanismos tecnológicos poseedores de diferentes características y aspectos importantes, su utilización conlleva a diferentes consecuencias favorables, como es el caso de una comunicación con mayor amplitud y la generación de interacción con otras personas sin importar el lugar donde se encuentre, solamente con el uso de la red Internet.

Manifiestamente se pudo identificar que el uso de las redes sociales está presente en los jóvenes en un alto nivel, determinando que su uso es trascendental para la comunicación existente entre los grupos sociales que se crean por medio de estas plataformas. Igualmente, esta situación se presenta a causa de la utilización del celular, por medio del cual se accede de manera inmediata a las aplicaciones siendo percibido como un generador de acceso rápido. Aunque existan diferentes redes sociales en la actualidad existen preferencias, no obstante, el uso de todas genera una conexión más pronta y así mismo determinada por ser oportunidades para la presencia de ataques en relación a la información depositada.

\section{REFERENCIAS}

Amazon. (2018). Logística de Amazon guía para principiantes. Building your Brand, 18 .

Argueta, R. A. (2018). Repositorio UASB. Obtenido de Marketing digital estrategias en redes sociales: http://repositorio.uasb.edu.ec

Banco Central de Colombia. (2019). Gobierno Digital Colombia. Obtenido de 
Estaditicas datos abiertos: https://www.datos.gov.co/ComercioIndustria-y-Turismo/

Barnes, R. L. (29 de enero de 2018). Invertir en Amazon y en su modelo de negocio horizontal. Obtenido de https://rodrigolbarnes.com/2018/01/29/mo delo-negocio-amazon/

Camuñas, E. (2017). Los beneficios de la investigación de mercados: claridad y acierto. UnirRevista.

Collins, P., Mockler, R. J., \& Gartenfeld, M. (2003). Amazon.com una tienda de comercio electrónico. Universidad de St. John's, 1-22.

Contreras, J. A. (2016). Repositorio UG. Obtenido de FACULTAD DE COMUNICACIÓN SOCIAL: http://repositorio.ug.edu.ec/bitstream/redug /18651/1/ULTIMOimprime.pdf

Cvijikj, I., \& Michahelles, F. (2013). Online engagement factors on Facebook brand pages. Social Network Analysis and Mining, 843-861.

de Vries, L., Gensler, S., \& Leeflang, P. (2012). Popularity of brand posts on brand fan pages: An investigation of the effects of social media marketing. Journal of Interactive Marketing, 26, 83-91.

Dolan, R., Conduit, J., Fahy, J., \& Goodman, S. (2016). Social media engagement behaviour: a uses and gratifications perspective. Journal of Strategic Marketing, 24, 261-277.

eShopex. (2018). Obtenido de Tarifas de Ecuador:

https://www.eshopex.com/ec/tarifas_infor macion.aspx

Feebo. (22 de marzo de 2016). Estudio de mercado: Amazon, el gigante del comercio electrónico. Obtenido de http://www.estudiosdemercadoonline.com/ estudio-mercado-amazon-gigante-delcomercio-electronico/

González, P. C. (2017 de noviembre de 11). $35 \%$ de los ecuatorianos compra por internet. El Comercio.

Inec. (2019). INEC. Obtenido de Instituto Nacional de Estadísticas y Censos: http://www.ecuadorencifras.gob.ec/estadist icas/

Lacort, J. (3 de septiembre de 2018). Así es como gana dinero Amazon: cada vez más nube $y$ un futuro de producciones audiovisuales. Recuperado el 8 de marzo de 2019, de Xataka: https://www.xataka.com

Lee, D., Hosanagar, K., \& Nair, H. (2018). The effect of advertising content on consumer engagement: Evidence from Facebook. Management Science.

Ludovic, J., Baghestan, A., \& Sazmand, Y. (2013). A Uses and Gratification Perspective on Social Media Usage and Online Marketing. Middle-East Journal of Scientific Research, 15, 134-145.

Meneses, T., \& Geilany, M. (13 de enero de 2017). Repositorio UCSG. Obtenido de Facultad de especialidades empresariales: http://repositorio.ucsg.edu.ec/bitstream/331 7/7841/3/T-UCSG-PRE-ESP-CIM-278.pdf

Morales, V. C. (2017). Repositorio UG. Obtenido de Facultad de comunicación social:

http://repositorio.ug.edu.ec/bitstream/redug /16144/1/CRISTHIAN\%20ANDR\%C3\%8 9S\%20MORALES\%20VERA.pdf

Muntinga, D., Moorman, M., \& Smit, E. (2011). Introducting COBRAs: Exploring motivations for brand-related social media use. International Journal of Advertising, 30, 13-46.

PASTOR, J. (14 de noviembre de 2018). Amazon lanza sus compras internacionales: 
compra todo en EE.UU., recíbelo en casa. Obtenido de https://www.xataka.com/

Przybylski, A. K., Murayama, K., DeHaan, C. R., \& Gladwell, V. (2013). Motivational, emotional, and behavioral correlates of fear of missing out. Computers in Human Behavior, 1814-1848.

Publigrata. (2017). 100 estadísticas claves de marketing digital 2017.

Publigrata. (s.f.). Marketing Digital Estudio de usos y hábitos Social media.

SENAE. (2019). Para Importar. Obtenido de ¿Cuánto se debe pagar en tributos por un producto importado?: https://www.aduana.gob.ec/para-importar/
Smock, A., Ellison, N., Lampe, C., \& Wohn, D. (2011). Facebook as a toolkit: A uses and gratification approach to unbundling feature use. Computers in Human Behavior, 27, 2322-2329.

Sun, S., Rubin, A., \& Haridakis, P. (2008). The Role of Motivation and Media Involvement in Explaining Internet Dependency. Journal of Broadcasting \& Electronic Media, 52, 408-431.

Trias, M. (2013). Ventas globales con Amazon. Amazon, 5.

Vera, A., Soto, G., \& Ortega, S. (2002). Estegias resumen de crecimiento horizontal y vertical: ¿unas a expensas de las otras? Investigaciones Europeas de Dirección y Economía de la Empresa, 181-198. 